\title{
Comprensión del discurso académico escrito en estudiantes de biología de la Universidad de Concepción ${ }^{1}$
}

\author{
Pamela Ramírez Peña* \\ Bernardo Riffo Ocares*
}

\begin{abstract}
RESUMEN
El presente artículo expone los resultados de una investigación de corte descriptivo realizada en la Universidad de Concepción con estudiantes en etapa inicial y terminal de la carrera de Biología en el ámbito de la comprensión del discurso especializado. La hipótesis de trabajo postula que el proceso de formación académica en las especialidades biológicas incide en el desarrollo de la comprensión de textos científicos en estudiantes universitarios, lo que se refleja principalmente en: a) diferencias en el nivel inferencial de lectura, b) en la percepción que los estudiantes tienen de los textos de su comunidad discursiva y c) en las estrategias que emplean al leer un texto científico, asociadas con el nivel de estudios universitarios de los sujetos. Para comprobar esta hipótesis se emplearon dos instrumentos: una prueba de lectura, compuesta por dos textos con distinto grado de especialización, y una encuesta aplicada a la muestra total. La prueba de lectura fue aplicada a una población de 57 individuos correspondientes a dos niveles de formación profesional y académica, mientras que la encuesta se aplicó a una población de 29 individuos divididos en los dos niveles antes mencionados. Los resultados permiten comprobar la hipótesis planteada y hacer visible una de las tareas pendientes del la Educación Superior: la necesidad de alfabetizar académicamente a los estudiantes que ingresan a la universidad.
\end{abstract}

Palabras clave: comprensión de lectura, textos especializados, comunidad discursiva, alfabetización académica.

\section{How biology students at the University of Concepcion understand written academic discourses}

\section{ABSTRACT}

This paper presents the results of a descriptive research at the University of Concepcion with students in the first and the last phases of Biology studies in the field of understanding the specialized discourse. The working hypothesis states that the process of academic training in biological specialties affects the development of the understanding of scientific texts in university students, which is mainly reflected in a) differences in reading inferential levels, b) the perception students have about the texts of their discourse community and c) the strategies they use when reading a scientific text in relation to their university level. In order

I Investigación realizada en el marco del desarrollo de la tesis conducente al grado académico de Magíster en Lingüística en la Universidad de Concepción.

* Magíster en Lingüística. Universidad de Concepción, Chile. Correo electrónico: pamerami@udec.cl

Doktor der Pbilosophie (Universität Bielefeld, Alemania). Universidad de Concepción, Chile. Correo electrónico: bernardo@udec.cl 
to demonstrate this hypothesis, two instruments were used, i.e. a reading test consisting of two texts with varying degrees of expertise and a survey applied to the total sample. The reading test was applied to a population of 57 individuals corresponding to two levels of vocational and academic training while the survey was applied to a population of 29 individuals divided into the two aforementioned levels. The results allow demonstrating the suggested hypothesis and reveal one of the remaining tasks of the higher education: the need to academically literate students who enter university.

Key words: Reading understanding, specialized texts, discourse community, academic literacy.

\section{Introducción}

El estudiante que ingresa a la Universidad debe manifestar el dominio de ciertas competencias que se relacionan con el éxito (o fracaso) académico: autonomía, responsabilidad, desarrollo de estrategias en función de objetivos de aprendizaje y un óptimo nivel de comprensión lectora, son algunas de ellas. De lo anterior se deduce que el proceso de formación académica requiere de un estudiante con ciertas competencias iniciales que, a medida que progrese en la formación disciplinar, irán mejorando y perfeccionándose en función de las demandas que imponen las prácticas discursivas propias de la comunidad discursiva. De esta forma, el proceso de formación académica genera cambios en los sujetos que como consecuencia producen la afiliación intelectual (Coulon, 1995) del estudiante con la comunidad. Pero ¿qué sucede durante el proceso de incorporación a la comunidad discursiva? ¿Cuáles son los cambios que se producen en los sujetos? Este trabajo intenta responder a estas preguntas enfocándose en uno de los aspectos centrales del proceso de enseñanza-aprendizaje: la comprensión lectora.

La comprensión lectora es un fenómeno complejo determinado por factores vinculados al lector (capacidad de memoria, factores atencionales, comportamiento estratégico), al texto (tipología, características lingüístico-discursivas) y al contexto en el cual se produce y circula un determinado género discursivo. A partir de lo anterior, esta investigación se origina considerando un enfoque interdisciplinario que permite dar cuenta del fenómeno 
de la comprensión de textos especializados desde una perspectiva ecológica e integral. Por ello, confluyen en este trabajo bases teóricas provenientes de la psicolingüística (modelo de construcciónintegración de Kintsch y Van Dijk, 1978) y de la teoría del género textual (Eggins, 2002; Parodi, 2008).

\section{Comprensión de textos especializados: un enfoque sociocognitivo}

Los factores involucrados en la comprensión de un texto especializado (lector, texto, contexto) permiten establecer tres dimensiones que interactúan constantemente durante el desarrollo del proceso de comprensión lectora. Estas dimensiones son: a) la dimensión cognitiva; b) la dimensión lingüística; y c) la dimensión contextual (Parodi, 2008).

a) Dimensión cognitiva: esta dimensión está centrada en el sujeto, quien lleva a cabo una serie de procesos mentales durante el proceso de comprensión lectora. En esta dimensión convergen los aportes de Kintsch y Van Dijk (1978) en lo que respecta a la teoría de la construcción-integración, la cual sostiene que el procesamiento de un texto se realiza en dos fases (construcción, integración), las cuales generan una representación semántica del texto en tres niveles: nivel de representación superficial, breve registro de la redacción en la memoria del lector; nivel de representación del texto base, especifica las relaciones semánticas entre los distintos componentes y partes del texto; y, el nivel de representación del modelo de situación, representación cognitiva análoga, no proposicional, de la situación referida en el texto. Para construir los niveles de representación señalados anteriormente, el lector debe desempeñar un rol activo, en función de la tarea de lectura, que le permita planificar, monitorear y evaluar el proceso de comprensión. Pero este enfoque no considera sólo los factores lingüísticos vinculados a la dimensión cognitiva, sino que también reconoce la importancia de otro concepto fundamental: el modelo de contexto (Van Dijk, 200I). De esta forma, el enfoque sociocognitivo considera que, en esta dimensión, el lector construye una representación mental de la 
situación referida en el texto y, además, reconoce la situación social en la que se produce el discurso, lo que implica reconocer los requerimientos que éste implica en cuanto a su recepción y comprensión. En otras palabras, el enfoque sociocognitivo sostiene que el lector que se ha incorporado a una comunidad discursiva determinada ha logrado construir una representación cognitiva del género disciplinar.

b) Dimensión lingüística: esta dimensión permite relacionar la dimensión cognitiva con la dimensión contextual, puesto que el lenguaje funciona como una herramienta que permite que lo que sucede en el mundo social alcance un estatus cognitivo. De este modo, el sujeto interactúa en un contexto específico y construye su realidad a través de la interacción con la comunidad discursiva en la cual se encuentra inmerso.

c) Dimensión contextual: esta dimensión está relacionada con el contexto cultural y situacional en el que se encuentra el sujeto. Este contexto se define en función de la disciplina, las Ciencias Biológicas en el caso de esta investigación, considerando los textos que habitualmente leen los estudiantes, las prácticas sociales de la comunidad, prácticas académicas específicas, entre otras. Constituye el nivel experiencial de este enfoque que es traducido a formatos lingüísticos para ser procesado hasta alcanzar un estatus cognitivo, produciéndose así un fenómeno denominado cognición situada (Parodi, 2008).

\section{La investigación}

\section{Objetivos}

a) Identificar las diferencias existentes en los procesos que subyacen a la comprensión del discurso académico escrito entre alumnos en etapa inicial de formación y etapa terminal de la carrera de Biología considerando el rendimiento por nivel textual, naturaleza de la información (implícita - explícita) y tipo de inferencia.

b) Conocer la percepción que docentes y estudiantes tienen de la comunidad discursiva en lo que respecta a manifestaciones textuales y prácticas de enseñanza - aprendizaje. 


\section{Hipótesis}

El proceso de formación académica en las especialidades biológicas incide en el desarrollo de la comprensión de textos científicos en estudiantes universitarios de pregrado de la carrera de Licenciatura en Biología, lo que se refleja principalmente en: a) diferencias en el nivel inferencial de lectura, b) en la percepción que los estudiantes tienen de los textos de su comunidad discursiva, y c) en las estrategias que emplean al leer un texto científico, asociadas con el nivel de estudios universitarios de los sujetos.

\section{Diseño}

Para comprobar la hipótesis planteada se llevó a cabo un estudio de campo de corte descriptivo que pretende dar cuenta del proceso de comprensión del discurso académico escrito que llevan a cabo estudiantes en etapa inicial y terminal de formación, desde el punto de vista cualitativo y cuantitativo. Se consideran además aspectos de tipo etnográfico que permiten conocer a la comunidad discursiva, tanto a los docentes como a los estudiantes de la carrera, seleccionar los textos para la elaboración de los instrumentos de evaluación, validar los instrumentos y, finalmente, elaborar las pautas de corrección de las pruebas.

\section{Procedimientos}

a) Población participante: la muestra para la prueba de lectura estuvo compuesta por 57 sujetos divididos en dos grupos: 43 sujetos con un nivel inicial de formación (dos o tres años de formación en la disciplina) y I4 sujetos con un nivel terminal de formación (cinco o más años de formación en la disciplina) (Figura I). 
Figura I: Distribución de la muestra de acuerdo al nivel de formación de los estudiantes

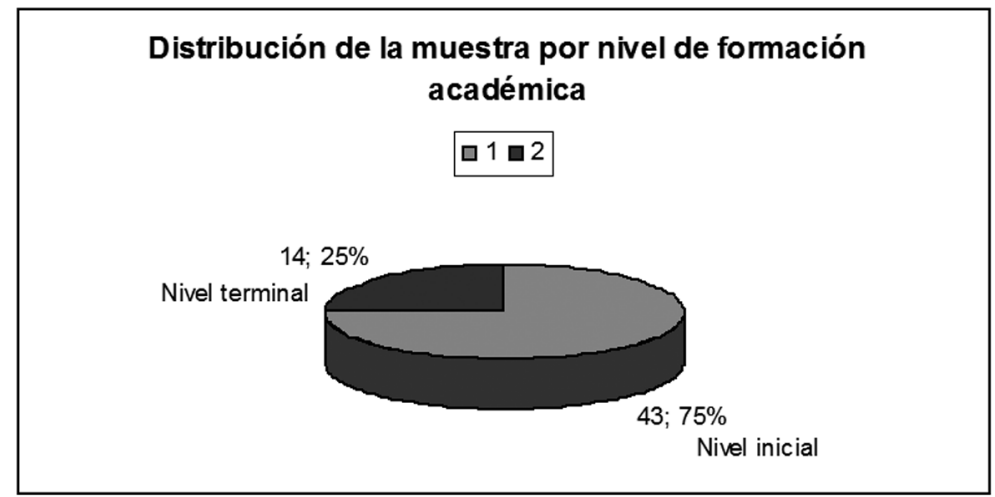

En la carrera de Biología, los estudiantes en general tardan más de 5 años en concluir las asignaturas que contempla la malla curricular, aun cuando el plan de estudios contempla IO semestres. Es por ello, que los sujetos de la muestra están agrupados en dos grupos, pero en cada grupo se observan diferencias en cuanto a los años de formación en la disciplina (Figura 2).

Figura 2: Años de formación académica de la muestra total.

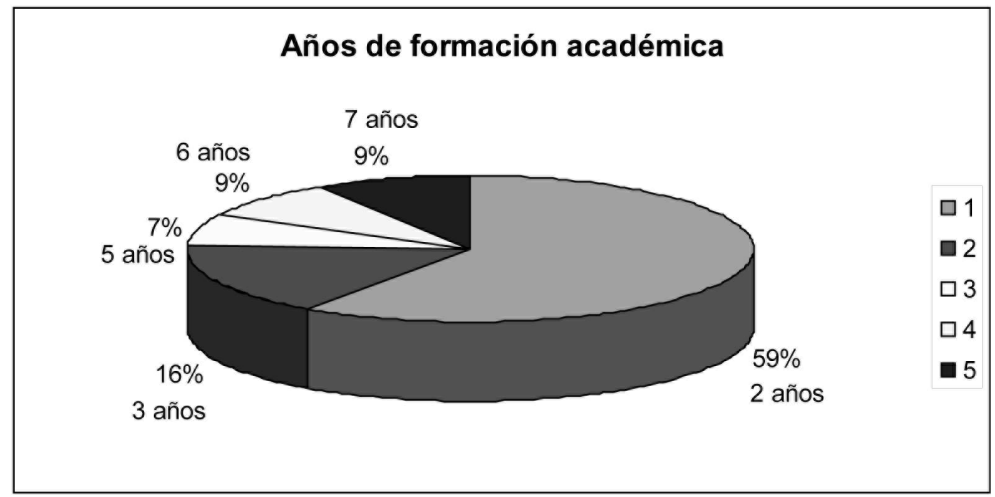

b) La prueba de lectura: para evaluar el nivel de comprensión de la muestra total se desarrolló un instrumento ad boc para la investigación: una prueba de lectura formada por un texto de divulgación científica y un artículo científico. La selección de los textos se realizó a partir del material que entregó uno de los docentes de la carrera. Para seleccionar aquellos que formarían parte 
de la prueba se consideró la frecuencia de lectura, la importancia que los docentes asignaron al tipo de texto dentro de la formación académica, y las características que definen un texto prototípico de la disciplina, como por ejemplo, ser un texto discontinuo, en el cual sea posible encontrar figuras explicativas, tablas y gráficos; presentar terminología propia del área y la densidad informativa, entre otras. El texto I, por ser de menor especialización, tenía menor presencia de terminología en comparación con el texto 2, y se empleaba una figura. El texto 2, en cambio, presentaba mayor densidad informativa, abundante uso de terminología y alusión al conocimiento previo. Además reforzaba la entrega de información a través de un gráfico y una tabla (TablaI)

Tabla I: Cuadro comparativo entre los textos empleados en la Prueba de Lectura

\begin{tabular}{|l|l|l|}
\hline & $\begin{array}{l}\text { TEXTO I } \\
\text { "Introducción a la teoría } \\
\text { de la evolución" }\end{array}$ & $\begin{array}{l}\text { TEXTO 2 } \\
\text { "Dieta de lycalopex griseus } \\
\text { (gray, I837) (mammalia: } \\
\text { canidae) en la depresión } \\
\text { intermedia del sur de } \\
\text { Chile." }\end{array}$ \\
\hline TIPO DE TEXTO & $\begin{array}{l}\text { Texto de divulgación } \\
\text { científica }\end{array}$ & Artículo científico \\
\hline $\begin{array}{l}\text { DENSIDAD } \\
\text { INFORMATIVA }\end{array}$ & Menor & Mayor \\
\hline $\begin{array}{l}\text { PRESENCIA DE } \\
\text { TERMINOLOGÍA }\end{array}$ & Menor & Mayor \\
\hline $\begin{array}{l}\text { RECURSOS } \\
\text { EMPLEADOS } \\
\text { (Formas discursivas, } \\
\text { otros recursos })\end{array}$ & $\begin{array}{l}\text { Predominio de la } \\
\text { enumeración descriptiva a } \\
\text { lo largo del texto } \\
\text { Presencia de una figura }\end{array}$ & $\begin{array}{l}\text { Predominio de secuencias } \\
\text { descriptivas y temporales. } \\
\text { Presencia de un gráfico } \\
\text { Presencia de una tabla } \\
\text { Presencia de fórmulas e } \\
\text { información porcentual }\end{array}$ \\
\hline
\end{tabular}

Para elaborar y clasificar las preguntas de forma que permita caracterizar la muestra en cuanto al proceso que los sujetos deben llevar a cabo para contestar, se utilizó como criterio de evaluación, la clasificación de tipo de preguntas planteada por Véliz y Riffo (I993). De esta forma las preguntas de la prueba se clasifican de la forma en que se muestra en la tabla siguiente (Tabla 2). 
Tabla 2: Clasificación de las preguntas de acuerdo al nivel de comprensión, tipo de pregunta (pregunta de respuesta explícita (PRE), pregunta de respuesta implícita (PRI)) y tipo de inferencia.

\begin{tabular}{|c|c|c|c|}
\hline $\begin{array}{c}\text { NIVEL DE } \\
\text { COMPRENSIÓN }\end{array}$ & $\begin{array}{c}\text { TIPO DE } \\
\text { PREGUNTA }\end{array}$ & $\begin{array}{c}\text { TIPO DE } \\
\text { INFERENCIA }\end{array}$ & $\begin{array}{c}\text { CANTIDAD DE } \\
\text { PREGUNTAS }\end{array}$ \\
\hline \multirow{4}{*}{ LOCAL } & PRI & Elaborativa & 2 \\
\cline { 2 - 4 } & PRE & Causalidad & I \\
\cline { 2 - 4 } & PRE & Espacial & I \\
\cline { 2 - 4 } & PRI & Informacional & I \\
\cline { 2 - 4 } & PRE & Informacional & I \\
\hline \multirow{5}{*}{ GLOBAL } & PRE & Elaborativa & I \\
\cline { 2 - 4 } & PRI & Elaborativa & I \\
\cline { 2 - 4 } & PRE & Causalidad & I \\
\cline { 2 - 4 } & PRI & Evaluativa & I \\
\cline { 2 - 4 } & PRE & Tema & Tema \\
\cline { 2 - 4 } & \multicolumn{2}{|c|}{ PRI } & I4 \\
\hline \multirow{2}{*}{ CANTIDAD TOTAL DE PREGUNTAS } & \multicolumn{2}{|c|}{} \\
\hline
\end{tabular}

El peso relativo de las preguntas de acuerdo al nivel de comprensión, tipo de pregunta y tipo de inferencia se observa claramente en los gráficos siguientes (Figuras 3, 4 y 5).

Figura 3: Distribución porcentual de preguntas que miden el nivel local y global de comprensión.

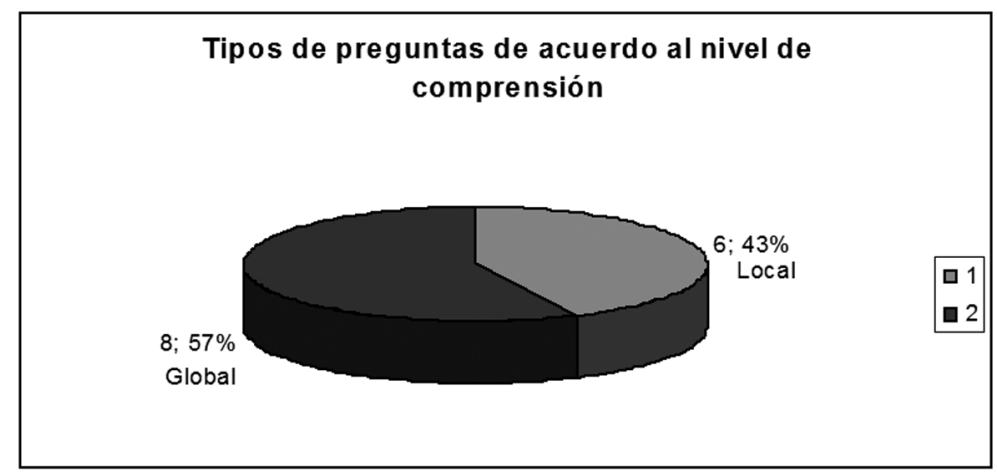


Figura 4: Distribución de preguntas basadas en información que se encuentra en la superficie textual (PRE) o preguntas cuyas respuestas se elaboran a partir del conocimiento previo o de pistas textuales (PRI).

\section{Tipo de pregunta de acuerdo a presencia de la información (implícita, explícita)}

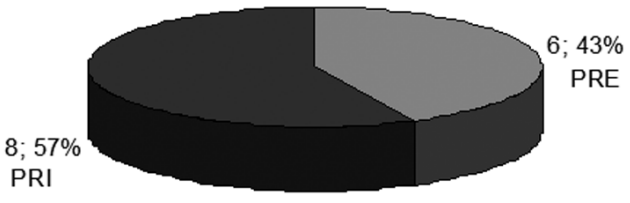

Figura 5: Distribución de preguntas según tipo de inferencia

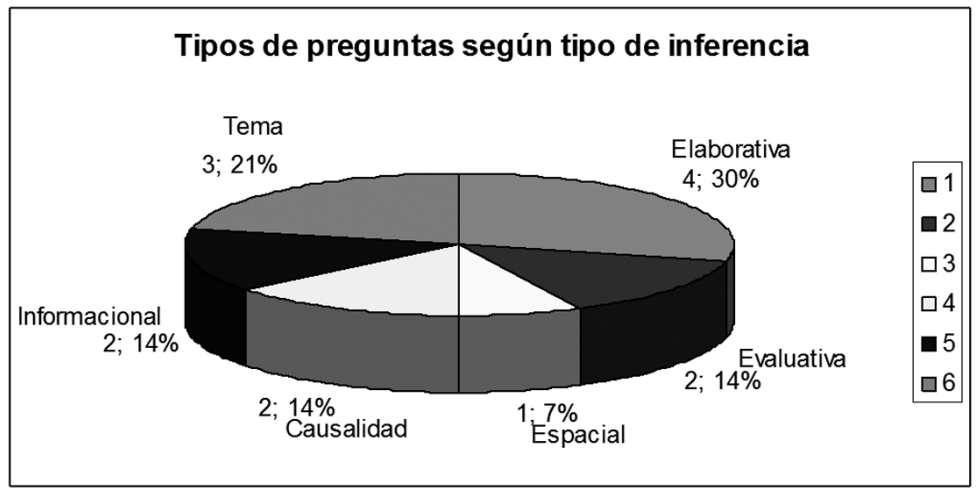

Las pruebas fueron validadas por los expertos ( 3 profesores) y la pauta de corrección fue realizada por una profesora de la carrera de Biología.

c) Aplicación y corrección de las pruebas: la aplicación de la prueba se desarrolló en una sesión por grupo en las salas de la facultad de Ciencias Naturales y Oceanográficas. Los estudiantes tenían una hora para leer el texto y contestar a las preguntas planteadas. Para la corrección de la prueba se asignó como puntaje máximo 2 puntos por respuesta (I punto respuesta parcialmente correcta). De esta forma, la prueba tenía un puntaje total de 28 puntos: I4 puntos para el texto I y I4 puntos para el texto 2 . 
d) Las encuestas: se aplicaron dos encuestas unos meses antes de la elaboración de la prueba de lectura vía correo electrónico, una de ellas destinada a los estudiantes y otra a los profesores. La encuesta destinada a los estudiantes se aplicó a un total de 29 individuos: I5 de primer año de formación y I4 de quinto año. Con la aplicación de este instrumento se buscó conocer las percepciones que los individuos tienen de los textos que leen y los procesos que llevan a cabo para comprender y aprender a partir de ellos. Para ello la encuesta se dividió en tres ítems: tipología textual, conocimiento previo y proceso de enseñanza - aprendizaje. La información que arroja este ítem permite conocer en forma superficial el comportamiento metacognitivo de los lectores.

La encuesta aplicada a los docentes tuvo como principal objetivo reunir información con respecto a la percepción que los docentes tienen de la forma en que sus alumnos aprenden a partir de un texto y la manera en que se lleva a cabo el proceso de enseñanza aprendizaje en la comunidad. Dos aspectos guiaron la elaboración de esta encuesta: por un lado, la percepción que los profesores tienen de sus alumnos con respecto a habilidades cognitivas de ingreso, nivel de conocimientos previos del estudiante que ingresa a la carrera, porcentaje y causas de deserción, habilidades cognitivas que definen al estudiante de quinto año. Por otro lado, esta encuesta buscaba también recabar información con respecto al proceso de enseñanzaaprendizaje en términos de gradación de textos, criterios de selección de los mismos, estrategias metodológicas empleadas por los docentes para evaluar el aprendizaje a partir de los textos de la disciplina, además de las habilidades cognitivas que los profesores esperan de sus estudiantes en relación con la comprensión de un texto del área de las Ciencias Biológicas.

\section{Resultados generales}

\section{Prueba de lectura}

Se realizó un análisis estadístico con el software InfoStat (2009). A partir de este análisis se obtienen los siguientes puntajes globales (máximos y mínimos) en la prueba de lectura (Tabla 4) 
Tabla 4: Resumen de los puntajes de los textos que constituyen la prueba por experiencia académica

\begin{tabular}{|l|c|c|c|c|c|c|}
\hline \multirow{2}{*}{ Experiencia Académica } & \multicolumn{3}{|c|}{ Puntaje Total Texto I } & \multicolumn{3}{|c|}{ Puntaje Total Texto 2 } \\
\cline { 2 - 6 } & $\mathrm{n}$ & Mín & Máx & Mín & Máx \\
\hline Inicial & 43 & 2 & I0 & 0 & I0 \\
\hline Terminal & I4 & 5 & I2 & 9 & I4 \\
\hline
\end{tabular}

La información que entrega la tabla indica que los estudiantes en etapa terminal de formación obtienen promedios de rendimiento significativamente mayores que los estudiantes que se encuentran en etapa inicial de formación en ambos textos. De esta forma, se establece una relación directamente proporcional entre experiencia académica y nivel de comprensión de un texto especializado, lo cual confirma la importancia que el proceso de formación académica tiene en la lectura de textos propios de la disciplina. Esta información se observa detalladamente en el siguiente gráfico (Figura 6).

Figura 6: Puntajes obtenidos para cada grupo en cada una de las evaluaciones.

Resultados Generales

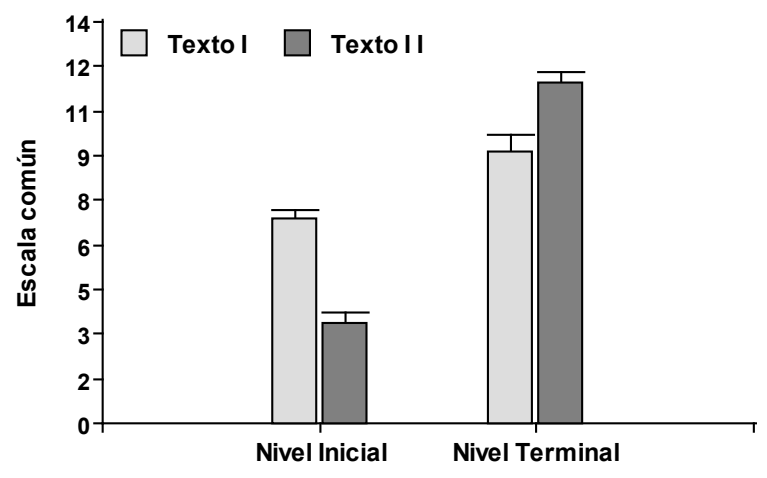

Experiencia Académica

La diferencia que se encontró en los rendimientos promedio de la muestra era previsible. Sin embargo, lo central en esta investigación fue determinar en qué aspectos del proceso de comprensión se encuentra la diferencia entre un grupo y otro. Tal como se postuló en la hipótesis, uno de los factores centrales asociados a esta diferencia es el procesamiento inferencial. Los resultados que arroja 
la investigación en relación con este aspecto de la comprensión indican lo siguiente:

a) La realización de inferencias elaborativas genera mayores dificultades en los estudiantes con menor experiencia académica. Esto indica que el sujeto carece de conocimientos previos que le permitan realizar este tipo de procesamiento, o, como se observó en algunas respuestas, no son capaces de reelaborar información que se encuentra en la superficie textual.

b) La realización de la inferencia causal obtiene uno de los menores rendimientos de la prueba, aun cuando se mantiene la diferencia que se produce entre los estudiantes en etapa inicial y terminal de formación. Con respecto a este tipo de inferencia, vale la pena señalar que la forma en que los sujetos elaboraron las respuestas a la pregunta fue muy distinta: mientras los iniciados en la disciplina copiaban literalmente la respuesta, lo que revela un procesamiento superficial del texto; los estudiantes en etapa terminal de formación parafrasearon la información proveniente del texto y la complementaron con información adicional, lo que demuestra la influencia de la experiencia académica al señalar información vinculada al tópico, pero no explícita en el texto.

Ambos resultados se observan en la siguiente figura:

Figura 7: Comparación de puntajes entre los niveles académicos

\section{Elaborativa y Causalidad}

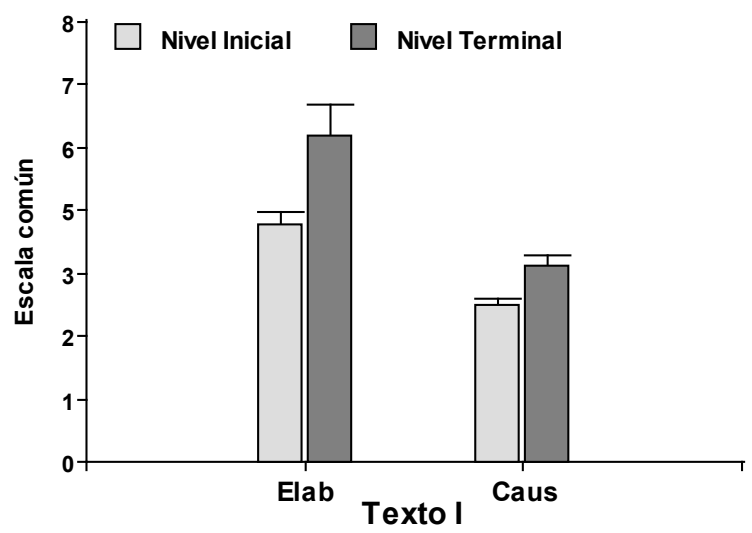


Los dos tipos de inferencia señalados anteriormente se consideraron en preguntas para medir el nivel de comprensión del texto de divulgación científica, por lo que el grado de especialización del mismo es bajo. Por ello, se esperaban mejores resultados para ambos grupos.

Las inferencias consideradas para la evaluación del artículo científico fueron: la inferencia espacial, informacional y temática. Los resultados obtenidos por la muestra indican lo siguiente:

c) Junto con la inferencia causal, la realización de la inferencia espacial obtiene también uno de los rendimientos más bajos. Esto podría reflejar una dificultad en la aplicación de macrorreglas (supresión, generalización, construcción) al momento de elaborar la respuesta, puesto que el sujeto debía seleccionar la información que permitiera nombrar y caracterizar el lugar donde se llevó a cabo la investigación que reporta el artículo. A pesar del bajo rendimiento que alcanza la muestra total, se mantiene la tendencia: el estudiante en etapa terminal de formación obtiene mejores resultados en la realización de este tipo de inferencia, lo que indica que estos sujetos son capaces de reconocer, localizar y sintetizar la información que exige la tarea.

d) Los rendimientos alcanzados por la muestra en la realización de inferencias informacionales indican que los estudiantes con mayor experiencia académica obtienen un nivel de rendimiento mayor que los estudiantes con menor experiencia académica. Esto podría estar determinado por la capacidad que tiene el estudiante en etapa terminal de formación de detectar pistas textuales y recurrir al conocimiento previo disciplinar. El rendimiento que obtiene el grupo en etapa inicial de formación es el de menor logro en la prueba de lectura, lo que confirma la correlación existente entre la experiencia académica y la comprensión de un texto especializado.

e) El rendimiento alcanzado por la muestra en la inferencia temática demuestra diferencias significativas entre ambos grupos: los estudiantes en etapa inicial de formación obtienen bajos resultados en la realización de este tipo de inferencia. Para evaluar el rendimiento en este nivel se les solicitó a los estudiantes que 
explicaran los datos de la investigación considerando una tabla y una figura (gráfico). Esta tarea permitió detectar que parte del grupo de estudiantes en etapa inicial de formación no reconoce la diferencia que existe entre una tabla y una figura, aun cuando cada elemento presentaba su denominación y explicación en la parte inferior. De lo anterior se deduce que los estudiantes con menor experiencia académica no están familiarizados con recursos característicos de textos altamente especializados debido a que no poseen una representación cognitiva del género discursivo, a diferencia de los estudiantes que poseen 5 o más años de formación en la disciplina.

Los resultados obtenidos por la muestra en las tres inferencias mencionadas anteriormente se observan en la siguiente figura:

Figura 8: Comparación de puntajes entre los niveles académicos.

\section{Inferencia Espacial e Informacional y Tema}

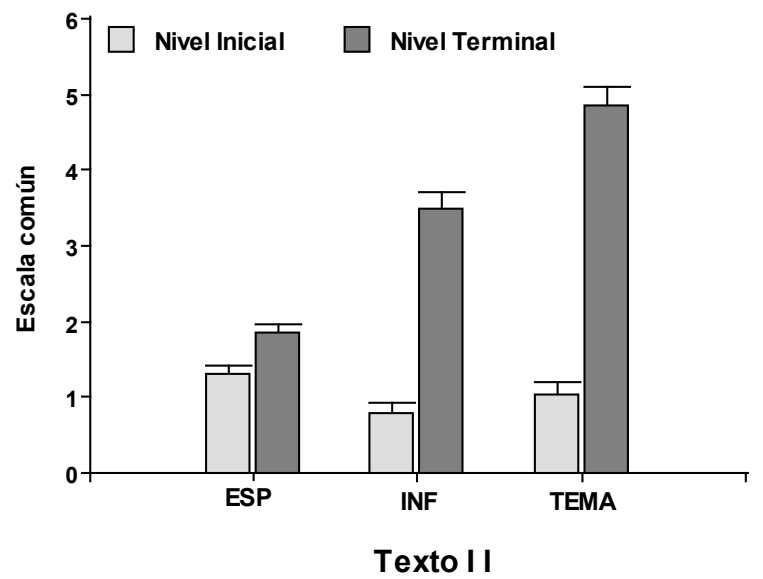

La realización de la inferencia evaluativa, que implica que el estudiante emita una opinión fundamentada con respecto a la información señalada en el texto, estuvo presente en ambos textos. Se esperaba que el rendimiento fuera similar en ambos grupos para el texto de divulgación científica debido al conocimiento que ambos tiene acerca del tema. Los resultados indican lo siguiente: 
f) La diferencia entre ambos grupos en la realización de la inferencia evaluativa en el texto de divulgación científica es el único valor no significativo con respecto a las diferencias en el nivel de rendimiento. Sin embargo, el rendimiento para este tipo de inferencia en el artículo científico demuestra diferencias significativas entre ambos grupos. De esta forma se comprueba la influencia de la experiencia académica debido a que el texto I requiere un nivel de conocimientos disciplinares básicos mientras que el texto 2 requiere de un dominio de conocimientos disciplinares vinculados al tema y al género discursivo. El nivel de rendimiento obtenido por la muestra se observa en la siguiente figura.

Figura 9: Comparación de puntajes entre los niveles académicos por texto

\section{Evaluativa}

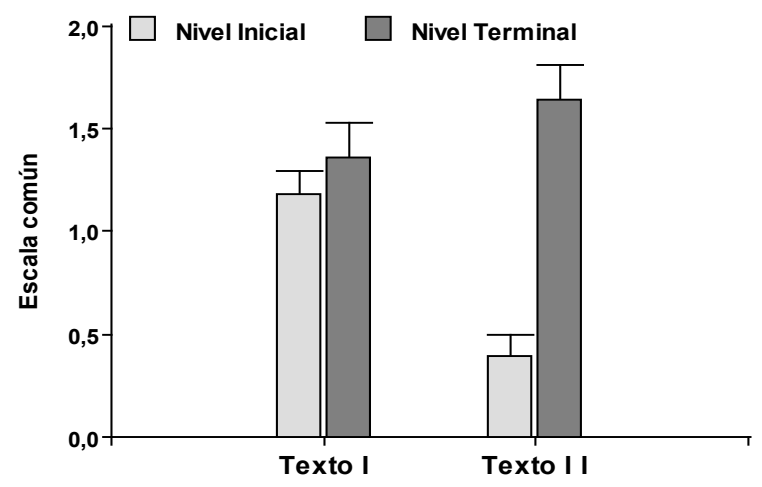

\section{Las encuestas}

Para analizar la información proveniente de las encuestas aplicadas a los estudiantes se creó una base de datos que fue analizada con el software estadístico SPSS I7 (2008). De esta forma se obtuvo un análisis estadístico simple con características descriptivas que, por medio de la observación de frecuencias y porcentajes, señala las distintas tendencias manifestadas en cada ítem por los sujetos del estudio. 
En términos generales, los datos indican que la percepción que los estudiantes tienen de los textos disciplinares cambia con el paso del tiempo en lo que respecta a los factores asociados a la mayor o menor complejidad del texto. La muestra coincide en señalar que los textos de la disciplina representan una complejidad media; sin embargo, los factores asociados a esta complejidad cambian en el transcurso del proceso de formación académica. Este cambio se debe a la exposición constante a distintas manifestaciones textuales representativas de la comunidad discursiva, lo que permite que el estudiante reconozca las características que presenta un texto propio de la disciplina, asigne la importancia que corresponde a cada elemento que forma parte del texto y aplique estrategias orientadas a obtener un mejor logro en la comprensión y aprendizaje a partir de un texto especializado. Por ejemplo, en lo que respecta a la presencia de terminología, los estudiantes en etapa inicial no asocian a una mayor complejidad la presencia de este recurso, mientras que los estudiantes en etapa terminal de formación asocian este factor a un grado de dificultad mayor, aunque esta frecuencia podría estar también relacionada con el grado de especialización de los textos que leen. En general, las frecuencias asociadas a las características textuales por las cuales se les pregunta a los estudiantes (disposición de ideas en el texto, extensión, densidad informativa, presencia de terminología) se observan con una dispersión mayor que en el caso de los estudiantes en etapa terminal de formación, lo cual indica que el estudiante no posee una representación cognitiva clara y precisa de los géneros discursivos que circulan en su comunidad, a diferencia del estudiante con 5 o más años de formación. He aquí otro indicador de la influencia que la experiencia académica tiene en la comprensión del discurso académico escrito, puesto que un lector que conoce y reconoce la superestructura textual y los recursos del género discursivo sabe cómo abordarlo para optimizar el nivel de comprensión del mismo.

La segunda parte de la encuesta consultaba a los estudiantes acerca de la frecuencia con que realizan ciertas estrategias de lectura (subrayar ideas principales, elaboración de resúmenes y esquemas, autoexplicación, crear y responder preguntas a partir del texto, complementar información). La información que arroja el análisis de las frecuencias indica que, en términos generales, las estrategias 
subrayar ideas principales y elaboración de resúmenes permanecen constantes a lo largo de tiempo, lo que indica que la experiencia académica no determina un cambio en la aplicación de éstas. Sin embargo, es de esperar que la calidad de las estrategias sí demuestre la influencia de la experiencia académica ${ }^{2}$. Las variaciones, que indican cambios en el sujeto en formación, se producen principalmente en la frecuencia de aplicación de las estrategias elaboración de esquemas, autoexplicación y crear - responder preguntas.

\section{Conclusiones}

El estudio realizado demuestra la incidencia que la experiencia académica tiene en la comprensión de un texto especializado. Dicha incidencia se observa con claridad en el rendimiento que alcanza la muestra en el nivel inferencial de lectura, puesto que la realización de inferencias informacionales, temáticas y elaborativas, principalmente, demuestran que el conocimiento previo disciplinar determina el nivel de comprensión lectora, toda vez que implica recurrir a conocimientos vinculados tanto al tópico como al género discursivo. De esta forma se comprueba que la representación cognitiva del género discursivo se construye con el paso del tiempo y tras la exposición constante a distintas manifestaciones textuales propias de la disciplina, lo que provoca también cambios en la percepción que el sujeto en formación tiene de los textos que lee $y$, por ende, cambios en su comportamiento estratégico. El sujeto que se inicia en la formación académica no distingue con claridad las características de los textos que lee, lo que se traduce en dificultades para abordar textos con distintos grados de especialización.

Para concluir, cabe señalar que la situación que se observa en la comunidad discursiva de la carrera de Biología comprueba que el proceso de afiliación intelectual es un proceso cognitivo y social muy complejo. Así lo demuestra el hecho de que sólo el I0\% de los estudiantes de la carrera egresan en el tiempo que estipula el plan de estudios. Para que este proceso genere mayores frutos, se hace

2 El resumen, como estrategia de lectura, fue estudiado por Iraís Hernández (2009) en la misma comunidad discursiva. 
necesario un proceso de alfabetización académica que permita nivelar a los estudiantes, disminuyendo la brecha que separa al estudiante ideal del estudiante real que comienza sus años de formación en la disciplina; en otras palabras, la comunidad discursiva que recibe a los novatos no debería actuar como si los sujetos que ingresan a la carrera contaran en forma natural con todas las habilidades que la disciplina exige, ya que la realidad ha demostrado que no es así.

\section{Bibliografía citada}

Carlino, P. (2005). Escribir, leer y aprender en la Universidad. Una introducción a la alfabetización académica. Buenos Aires, Argentina: F.C.E.

Coulon, A. (1995). Etnometodología y educación. En Tapia et al. (2003). Aplicación de una pauta diseñada para evaluar informes académicos universitarios. Rev. Signos vol. 36, No. 54, 249-257.

Eggins, S. (2002). Introducción a la lingüística sistémica. Logroño: Universidad de La Rioja.

Hernández, I. (2009). La comprensión discursiva de tres subgéneros académicos en una muestra estratificada de la comunidad de biólogos. Tesis doctoral. Universidad de Concepción, Chile.

Kintsch, W. (1988). The Role of Knowledge in Discourse Comprehension: A Construction Integration Model, Psychological Review, 95, I63-I82.

Kintsch, W. (1998). Comprehension: A paradigm for cognition. Cambridge: Cambridge University Press.

Kintsch, W. y Van Dijk, T. (I978). Toward a model of text comprehension and production. Psychological Review, vol. 85, 363-394.

Parodi, G. (2008). Géneros Académicos y Géneros Profesionales: Accesos Discursivos para Saber y Hacer. Valparaíso, Chile: Ediciones Universitarias de Valparaíso.

Van Dijk, T. (200I). Algunos principios de una teoría del contexto. En ALED. Revista latinoamericana de estudios del discurso, vol I(I), 69-8I. Disponible en: http://www.discursos.org/Art/Algunos\%20principios $\% 20$ de $\% 20$ una\%20teor\%EDa\%20de1\%20contexto.pdf

Véliz, M. y Riffo, B. (I993). Comprensión textual: criterios para su evaluación. RLA Vol. 31, I63-190. 